\section{Successful plasmapheresis in the Miller-Fisher syndrome}

The Miller-Fisher variant of acute idiopathic polyneuropathy (Landry-Guillain-Barré syndrome) is characterised by external ophthalmoplegia, ataxia, and areflexia. ${ }^{1}$ Though the syndrome is usually benign, progressive motor and respiratory impairment necessitating ventilatory support has been reported. ${ }^{2}$ The anatomical basis of this condition and its relation to the Landry-Guillain-Barré syndrome is contentious. ${ }^{3}$ We report a case with clinical and radiological evidence of a cerebellar parenchymal lesion and progressive respiratory impairment that responded appreciably within six hours of plasmapheresis on day 8 of the illness.

\section{Case report}

A previously well 14-year-old boy presented two weeks after a mild respiratory illness with a two-day history of diplopia and ataxia. Examination showed minimal external ophthalmoplegia, bilateral mydriasis, prominent horizontal phasic nystagmus on abduction, vertical nystagmus on supraduction, and truncal ataxia. All deep tendon reflexes were absent despite Jendrassik reinforcement, and there was minimal weakness in the limbs. Sensory modalities were intact apart from vibration sense, which was lost to the level of the sternum. By day 8 he had developed complete iridoplegia and ophthalmoplegia, including ptosis, and respiratory vital capacity had fallen to 1.41 . He could not walk unaided owing to severe ataxia. Autonomic instability, including arterial hypertension, was present.

Cerebrospinal fluid protein on day 4 was $1.45 \mathrm{~g} / 1$ and the $\mathrm{IgG}$ :protein ratio normal. Cerebrospinal fluid contained no significant cells, and the venereal disease research laboratory test was negative. Routine haematological and biochemical variables were normal. A computerised brain scan with contrast injection on day 3 showed a small low-density area in the left cerebellar hemisphere, which was absent on repeat scanning at seven weeks. Electromyography and nerve conduction studies including upper limb sensory and motor M- and F-wave latencies were performed on days 10 and 17 and were normal.

Treatment consisted of four sessions of plasmapheresis totalling 6.41 on days $8,9,10$, and 11 . Vital capacity increased to $2 \cdot 51$ after the first session, fell to $2 \cdot 21$ the following morning, and rose to 2.91 after the second session (figure). Pupillary response to light and minimal horizontal eye movements were detected after the second session. Six days after the start of plasmapheresis abduction was $45^{\circ}$ and infraduction complete. Pupillary responses were normal, and he walked unaided with a wide-based gait. Biceps reflexes were detectable on day 20 . He was fully recovered at eight weeks.

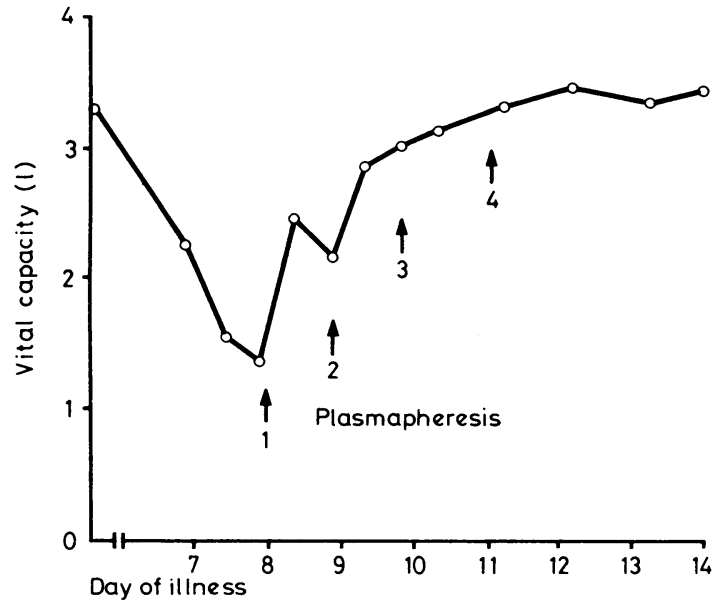

Respiratory vital capacity related to sessions of plasmapheresis.

\section{Comment}

Plasmapheresis has been increasingly reported to be of value in acute idiopathic polyneuropathy and chronic progressive demyelinating polyneuropathy since it was first suggested in 1978.4 The immunopathology of these conditions is not completely understood and the beneficial action of plasmapheresis not clear.
On day 8 of our patient's illness respiratory vital capacity fell to 1.41 and was accompanied by complete ophthalmoplegia and iridoplegia. Four sessions of plasmapheresis totalling 6.41 were conducted on four successive days. A dramatic improvement in vital capacity (figure) followed the first session and was subsequently maintained. The ocular disorder recovered within days but truncal ataxia persisted for six weeks. A recent report of a contrast-enhancing lesion in the midbrain tegmentum seen on computerised brain scanning in a patient with the Miller-Fisher syndrome is of interest. ${ }^{5}$ The initial scan in our patient showed a low-density area in the left cerebrellar hemisphere, but interpretation in this region is notoriously difficult. Prominent vertical and horizontal nystagmus early in the illness suggests a central parenchymal lesion.

The clinical course of acute idiopathic polyneuropathy and the Miller-Fisher variant is extremely varied. We are unaware of reports of plasmapheresis in the Miller-Fisher syndrome. The progressive deterioration in ventilatory and oculomotor function in our patient, followed by dramatic respiratory improvement within hours of plasmapheresis, suggests a role for this treatment in complicated Miller-Fisher syndrome. Future confirmation of a parenchymal demyelinating component in this condition may have implications for plasmapheresis in other demyelinating disorders of the nervous system.

We thank Dr $\mathbf{R}$ Herman, department of haematology, Royal Perth Hospital, for supervising the plasmapheresis.

${ }^{1}$ Fisher M. Unusual variant of acute idiopathic polyneuritis (syndrome of ophthalmoplegia, ataxia and areflexia). $N$ Engl $\mathcal{F}$ Med 1956;255:57-75.

${ }^{2}$ Blau I, Casson I, Lieberman A, Weiss E. The not so benign Miller Fisher syndrome. Arch Neurol 1980;37:384-5.

${ }^{3}$ Carroll W, Mastaglia F. Ocular involvement in post infective polyneuropathy. Clinical and Experimental Neurology 1977;14:66-74.

${ }^{4}$ Brettle RP, Gross M, Legg NJ, Lockwood M, Pallis C. Treatment of acute polyneuropathy by plasma exchange. Lancet 1978;ii:1100.

5 Derakhshan I, Lotfi J, Kaufman B. Ophthalmoplegia, ataxia, and hyporeflexia (Fisher's syndrome). Eur Neurol 1979;18:361-6.

(Accepted 2 December 1980)

Department of Neurology and Clinical Neurophysiology, Fremantle Hospital, Fremantle 6160, Western Australia

ROSS LITTLEWOOD, MB, BS, resident in neurology SERGE BAJADA, MB, FRACP, consultant neurologist

\section{Continuous subcutaneous insulin infusion corrects exercise-induced albuminuria in juvenile diabetes}

Juvenile-onset diabetes treated with insulin is characterised by either a normal ${ }^{1}$ or slightly raised $^{2}$ urinary albumin concentration as measured at rest. Viberti et al reported a reduced increase in urinary albumin excretion when insulin treatment was changed from conventional injections to continuous subcutaneous infusion. ${ }^{2}$ Patients with normal urinary albumin excretion at rest, however, may excrete increased amounts of albumin during exercise. ${ }^{3}$ Hence values obtained after exercise may be a more sensitive measure of abnormal renal handling of albumin than values obtained at rest. ${ }^{3}$ We report correction of exercise-induced albuminuria after two weeks of continuous subcutaneous insulin infusion in patients with juvenileonset diabetes.

\section{Patients, methods, and results}

We studied 10 insulin-dependent diabetic men aged 19 to 40 years (mean age $27 \pm$ SEM 2 years, mean weight $70 \pm 2 \mathrm{~kg}$, mean height $179 \pm 2 \mathrm{~cm}$ ). They were known to have had diabetes for two to 19 years (mean $10 \pm 2$ years) and were taking 28 to 74 units (mean $53+4 \mathrm{U}$ ) of intermediate-acting or short-acting and intermediate-acting insulin daily. There was no clinical 